\title{
A Measurement of the Spatial Distribution of Diffuse TeV Gamma Ray Emission from the Galactic Plane with Milagro
}

\author{
A. A. Abdo, ${ }^{1}$ B. Allen,${ }^{2}$ T. Aune, ${ }^{3}$ D. Berley,${ }^{4}$ E. Blaufuss ${ }^{4}$ S. Casanova, ${ }^{5}$ C. Chen,${ }^{6}$ \\ B. L. Dingus, ${ }^{7}$ R. W. Ellsworth,${ }^{8}$ L. Fleysher, ${ }^{9}$ R. Fleysher, ${ }^{9}$ M. M. Gonzalez,${ }^{10}$ \\ J. A. Goodman, ${ }^{4}$ C. M. Hoffman, ${ }^{7}$ P. H. Hüntemeyer, ${ }^{7}$ B. E. Kolterman,${ }^{9}$ C. P. Lansdell,${ }^{11}$ \\ J. T. Linnemann, ${ }^{12}$ J. E. McEnery, ${ }^{13}$ A. I. Mincer, ${ }^{9}$ I. V. Moskalenko, ${ }^{14}$ P. Nemethy,${ }^{9}$ \\ D. Noyes, ${ }^{4}$ T. A. Porter, ${ }^{3}$ J. Pretz,${ }^{7}$ J. M. Ryan,,${ }^{15}$ P. M. Saz Parkinson, ${ }^{3}$ A. Shoup,${ }^{16}$ \\ G. Sinnis, ${ }^{7}$ A. J. Smith,${ }^{4}$ A. W. Strong, ${ }^{17}$ G. W. Sullivan, ${ }^{4}$ V. Vasileiou, ${ }^{4}$ G. P. Walker,${ }^{7}$ \\ D. A. Williams, ${ }^{3}$ and G. B. Yodh ${ }^{6}$
}

\begin{abstract}
Diffuse $\gamma$-ray emission produced by the interaction of cosmic-ray particles with matter and radiation in the Galaxy can be used to probe the distribution
\end{abstract}

\footnotetext{
${ }^{1}$ Naval Research Laboratory, Washington, DC.

${ }^{2}$ Harvard-Smithsonian Center for Astrophysics, Cambridge, MA.

${ }^{3}$ University of California, Santa Cruz, CA.

${ }^{4}$ University of Maryland, College Park, MD.

${ }^{5}$ Max-Planck-Institut für Kernphysik, Heidelberg, Germany.

${ }^{6}$ University of California, Irvine, CA.

${ }^{7}$ Los Alamos National Laboratory, Los Alamos, NM.

${ }^{8}$ George Mason University, Fairfax, VA.

${ }^{9}$ New York University, New York, NY.

${ }^{10}$ Instituto de Astronomía, Universidad Nacional Autónoma de México, D.F., México.

${ }^{11}$ Institute for Defense Analyses, Alexandria, VA.

${ }^{12}$ Michigan State University, East Lansing, MI.

${ }^{13}$ NASA Goddard Space Flight Center, Greenbelt, MD.

${ }^{14}$ HEPL \& KIPAC, Stanford University, Stanford, CA.

${ }^{15}$ University of New Hampshire, Durham, NH.

${ }^{16}$ Ohio State University, Lima, OH.

${ }^{17}$ Max-Planck-Institut für extraterrestrische Physik, Garching, Germany.
} 
of cosmic rays and their sources in different regions of the Galaxy. With its large field of view and long observation time, the Milagro Gamma Ray Observatory is an ideal instrument for surveying large regions of the Northern Hemisphere sky and for detecting diffuse $\gamma$-ray emission at very high energies. Here, the spatial distribution and the flux of the diffuse $\gamma$-ray emission in the $\mathrm{TeV}$ energy range with a median energy of $15 \mathrm{TeV}$ for Galactic longitudes between $30^{\circ}$ and $110^{\circ}$ and between $136^{\circ}$ and $216^{\circ}$ and for Galactic latitudes between $-10^{\circ}$ and $10^{\circ}$ are determined. The measured fluxes are consistent with predictions of the GALPROP model everywhere except for the Cygnus region $\left(l \in\left[65^{\circ}, 85^{\circ}\right]\right)$. For the Cygnus region, the flux is twice the predicted value. This excess can be explained by the presence of active cosmic ray sources accelerating hadrons which interact with the local dense interstellar medium and produce gamma rays through pion decay.

Subject headings: gamma rays: observations

\section{Introduction}

One hundred years after their discovery, the origin, acceleration, and propagation of Galactic cosmic rays is still unclear. Supernova remnants (SNRs) and pulsars are the preferred candidate sources of Galactic cosmic rays. The detection of $\mathrm{TeV} \gamma$ rays and X-rays from the same locations within SNRs provides strong evidence that electrons are accelerated

in SNRs (Aharonian et al. 2006a). However, no compelling evidence for the acceleration of hadrons in SNRs has yet been found. The Galactic diffuse $\gamma$-ray emission originates from the interactions of cosmic rays (hadrons and electrons) with the matter and radiation fields in the Galaxy. Cosmic ray hadrons interact with matter producing neutral pions which in turn decay into $\gamma$ rays while cosmic-ray electrons produce $\mathrm{TeV} \gamma$ rays by inverse Compton (IC) scattering off the interstellar radiation fields. Therefore, $\gamma$ rays can provide information about the density and spectra of cosmic rays throughout the Galaxy. Gamma rays above $10 \mathrm{TeV}$, produced by the highest energy particles accelerated in SNR shocks, can be used to probe the acceleration processes in Galactic sources. The spatial distribution of these $\mathrm{TeV}$ $\gamma$ rays can be compared to model predictions of the diffuse $\gamma$-ray production from $\pi^{0}$ decays and IC scattering and thus the relative contribution of hadronic and leptonic mechanisms can be investigated.

EGRET data on the diffuse emission from the Galactic plane (Hunter et al. 1997) show a harder $\gamma$-ray spectrum from the inner Galaxy than predicted on the basis of the cosmic-ray spectrum and intensity measured at Earth (Bertsch et al. 1993; Strong et al. 
2004a,b). Many explanations for this "GeV excess" have been proposed, including a harder Galactic proton or electron spectrum (Gralewicz et al. 1997; Aharonian \& Atoyan 2000; Strong et al. 2004a,b; Porter \& Protheroe 1997) and the annihilation of dark matter particles (de Boer et al. 2005). Recent studies of the EGRET data have also investigated experimental uncertainties associated with the determination of the Galactic diffuse emission and are discussed in Moskalenko et al. (2007a). Stecker et al. (2008) concluded that the EGRET sensitivity above $1 \mathrm{GeV}$ may have been overestimated, while Baughman et al. (2007) concluded it may have been underestimated, leaving the situation unclear.

The first measurement of diffuse emission above $3.5 \mathrm{TeV}$ from a large region of the Galactic plane (Galactic longitudes $40^{\circ}<l<100^{\circ}$ ) indicated the existence of a $\mathrm{TeV}$ excess (Atkins et al. 2005; Prodanović et al. 2007; Casanova \& Dingus 2007). More recent measurements of the diffuse emission near $12 \mathrm{TeV}$ from the Cygnus region of the Galaxy (Abdo et al. 2007a) also show an excess when compared to predictions of GALPROP, a numerical model of cosmic-ray propagation in the Galaxy. Recently, HESS has detected very high-energy (VHE) diffuse emission from the Galactic Center Ridge, that is correlated with giant molecular clouds. The spectrum of the diffuse emission from the Galactic Center Ridge is significantly harder than the spectrum of the diffuse emission predicted by assuming the local cosmic-ray spectrum (Aharonian et al. 2006b). These previous results from Milagro and HESS support the hypothesis that the cosmic-ray flux is likely to vary throughout the Galaxy.

The Milagro (Atkins et al. 2004) experiment is a water-Cherenkov detector at an altitude of $2630 \mathrm{~m}$. It is composed of a central $60 \mathrm{~m} \times 80 \mathrm{~m}$ pond with a sparse $200 \mathrm{~m} \times 200 \mathrm{~m}$ array of 175 "outrigger" tanks surrounding it. The pond is instrumented with two layers of photomultiplier tubes. The top, "air-shower", layer consists of 450 PMTs under $1.4 \mathrm{~m}$ of water while the bottom, "muon", layer has 273 PMTs located $6 \mathrm{~m}$ below the surface. The air-shower layer allows the accurate measurement of shower particle arrival times used for direction reconstruction and triggering. The greater depth of the muon layer is used to detect penetrating muons and hadrons. The outrigger array, added in 2003, improved the angular resolution of the detector from $\sim 0.75^{\circ}$ to $\sim 0.45^{\circ}$ by providing a longer lever arm with which to reconstruct events. Milagro's large field of view $(\sim 2 \mathrm{sr})$ and high duty cycle $(>90 \%)$ allow it to monitor the entire overhead sky continuously, making it well-suited to measuring diffuse emission.

Here, the Milagro measurement of the diffuse emission around $15 \mathrm{TeV}$ from a region of the Galactic plane of longitudes $l \in\left[30^{\circ}, 110^{\circ}\right]$ and $l \in\left[136^{\circ}, 216^{\circ}\right]$, and latitudes $b \in\left[-10^{\circ}, 10^{\circ}\right]$ is presented. The measured $\gamma$-ray flux and the latitudinal and longitudinal profiles of the emission are reported and compared to predictions of the GALPROP 
model (Strong et al. 2000, 2004a, b; Porter et al. 2008). In GALPROP, first the propagation of cosmic rays in the Galaxy is modeled, and then the $\gamma$-ray emissivities are calculated using the propagated spectra of cosmic rays and the gas and radiation densities. The "conventional" model is tuned to reproduce the local direct cosmic-ray measurements. The "optimized" model has been designed to reproduce the EGRET data by relaxing the restriction from the local cosmic-ray measurements. In this version of the model, the proton spectrum is constrained by the cosmic-ray antiproton measurements and the electron spectrum is constrained using the EGRET data themselves.

Below, the course of the analysis is described, followed by the presentation of the results and a comparison with GALPROP predictions. In the last section, likely interpretations of the observations are discussed.

\section{Analysis}

The Milagro data, collected between July 2000 and November 2007, were analyzed using the method described in Abdo et al. (2007a). Only events with a zenith angle less than $45^{\circ}$ are included, which corresponds to declinations between $-7^{\circ}$ and $81^{\circ}$. The event excess is calculated using the background estimation method described in Atkins et al. (2003) with the modification that the events are weighted by a factor dependent on the $\gamma$-hadron separation parameter $A_{4}$ (Abdo et al. 2007a). Only declinations $<70^{\circ}$ are considered. This choice is governed by the fact that for $\delta>70^{\circ}$ the Galactic equator turns parallel to the right ascension axis. This causes the ratio of on- to off-time in the background calculation (Atkins et al. 2003) to become too big for signal bin sizes of 2 deg longitude by 4 deg latitude which is the bin size that is used in the Galactic longitude flux profile. As a result, the present analysis is insensitive for $\delta>70^{\circ}$ or $l \in\left[111^{\circ}, 135^{\circ}\right]$.

Within the region studied here, Milagro has previously detected four sources and four source candidates (Abdo et al. 2007a,b). The contribution from these sources is taken into account by modeling each source as a two-dimensional Gaussian plus a constant. The source location $(R A, \delta)$, the amplitude and radial width of the Gaussian, and the constant are determined using a $\chi^{2}$ minimization. The excess from each source is then calculated bin by bin using the resulting Gaussian function and subtracted from the total excess in the $0.1^{\circ} \times 0.1^{\circ}$ bin event excess map of the Galactic plane. The resulting diffuse event excess is converted to a flux with a Monte Carlo simulation of extensive air showers (CORSIKA, Heck et al. (1998)) and of the Milagro detector (GEANT4, Agostinelli et al. (2003)). The diffuse flux is calculated assuming a power law photon spectrum with a differential spectral index $\alpha=-2.75$. This spectral index was chosen to match the cosmic-ray spectrum in the energy range of this 
analysis (around $10 \mathrm{TeV}$ ). For a spectral index of -2.75 the median energy of detected events used in this analysis is $15 \mathrm{TeV}$.

Studies of possible sources for systematic errors have been performed. The size of the fit region around the eight sources and source candidates was varied. The Gaussian fits to the event excesses were performed in boxes centered around the sources of $4^{\circ} \times 4^{\circ}$, $6^{\circ} \times 6^{\circ}$, and $8^{\circ} \times 8^{\circ}$. The flux determination was also repeated for spectral indices of -2.4 and -2.9. The variations of the calculated fluxes were found to be less than $18 \%$. Another study concerned the background estimation. The background is estimated using the method of direct integration, calculating a background map using events over a $2 \mathrm{hr}$ time interval (Atkins et al. 2003). To account for possible contamination of the background from signal events two prominent regions in the Milagro sky map are excluded when doing this: a 2 by 2 degree region around the Crab Nebula location and a \pm 2.5 degree region around the Galactic plane. To investigate possible systematic effects in the background estimation the size of the region of exclusion around the Galactic plane was increased to \pm 5 degrees. The flux variations were found to be less than $7 \%$. These systematic errors are added in quadrature to a $30 \%$ systematic error which is derived from the underestimation of the Milagro trigger rate by cosmic ray shower simulations (Abdo et al. 2007a).

\section{Results}


Table 1. Gamma-ray emission from the Galactic plane around $15 \mathrm{TeV}$.

\begin{tabular}{|c|c|c|c|c|}
\hline \multirow{2}{*}{$\begin{array}{l}\text { Region } \\
\text { for }|b|<\mathbf{2}^{\mathbf{0}} \\
\quad(1, \mathrm{deg})\end{array}$} & \multirow{2}{*}{$\begin{array}{c}\text { Statistical } \\
\text { Significance } \\
\sigma\end{array}$} & \multicolumn{3}{|c|}{$\begin{array}{c}\text { Diffuse Flux }\left(\times 10^{-13} \mathrm{TeV}^{-1} \mathrm{~cm}^{-2} \mathrm{~s}^{-1} \mathrm{sr}^{-1}\right) \\
\text { GALPROP }\end{array}$} \\
\hline & & Milagro $^{\mathrm{a}}$ & optimized & conventional \\
\hline $30<l<65$ & 5.1 & $23.1 \pm 4.5_{-8.0}^{+7.0}$ & 20.0 & 4.9 \\
\hline $65<l<85$ & 8.6 & $21.8 \pm 2.5_{-7.8}^{+7.2}$ & 10.2 & 2.7 \\
\hline $85<l<110$ & 1.3 & $<7.1$ (95\% c.l.) & 5.8 & 1.3 \\
\hline $136<l<216$ & 0.8 & $<5.7$ (95\% c.l.) & 3.1 & 0.9 \\
\hline
\end{tabular}

aThe first error represents the statistical, the second the systematic uncertainty. See text for details. 
The upper plot in Figure 1 shows the Galactic longitude profile of the $\gamma$-ray emission as measured by Milagro before and after source contributions are subtracted. A $\gamma$-ray flux increase towards the Galactic center is visible, as well as the Cygnus region $\left(l \in\left[65^{\circ}, 85^{\circ}\right]\right)$ with a "bump" in the flux profile, even after source contributions are subtracted. The sourcesubtracted data points in the lower plot of Figure 1 are overlaid with the $\gamma$-ray emission profiles as predicted by the optimized GALPROP model, version 53_6102129RG (Strong et al. 2004a, b; Porter et al. 2008). The most significant discrepancy between model predictions and data appears in the Cygnus region. Table 1 lists the emission in four different regions with the Cygnus region being the most significant, $8.6 \sigma$ above the background. The predictions of the optimized and conventional GALPROP model are also given in Table 1. The measured diffuse flux from the Cygnus region is two (eight) times higher than the optimized (conventional) GALPROP prediction. The measured flux from the inner Galaxy $\left(l \in\left[30^{\circ}, 65^{\circ}\right]\right)$ is consistent with the optimized model and about five times higher than predicted by the conventional model. Calculating the ratio of the flux measured in the inner Galaxy to the flux measured in the Cygnus region cancels systematic detector effects like the underestimation of the trigger rate. The flux ratio in the data is calculated to be $1.1 \pm 0.2$ (stat.) compared to a ratio of 2.0 predicted by GALPROP. For the regions $l \in\left[85^{\circ}, 110^{\circ}\right]$ and $l \in\left[136^{\circ}, 216^{\circ}\right]$, flux upper limits are quoted since the statistical significances above the background are less than two standard deviations.

The energy spectra as predicted by the optimized GALPROP model in the Cygnus region and in the inner Galaxy, $l \in\left[30^{\circ}, 65^{\circ}\right]$, are shown in Figure 2 together with the EGRET and Milagro measurements. As can be seen, at Milagro energies the dominating GALPROP contribution to the diffuse flux is due to inverse Compton scattering of cosmicray electrons off the cosmic microwave background (CMB). The electron injection spectrum of the optimized GALPROP model is chosen such that the diffuse $\gamma$-ray emission spectrum matches the EGRET measurement at GeV energies. The injection spectrum is a broken power law with a spectral index of -1.5 below $20 \mathrm{GeV}$ and a spectral index of -2.42 above $20 \mathrm{GeV}$ extending to a maximum electron energy of $1000 \mathrm{TeV}$ with an electron flux of $1.4 \times 10^{-9} \mathrm{MeV}^{-1} \mathrm{~cm}^{-2} \mathrm{~s}^{-1} \mathrm{sr}^{-1}$ at $34.5 \mathrm{GeV}$ (Strong et al. $\left.2004 \mathrm{a}, \mathrm{b}\right)$ ).

Figure 3 shows the Galactic latitude profiles for $b \in\left[-10^{\circ}, 10^{\circ}\right]$ in three regions between Galactic longitude $l=30$ and $l=110$. Gaussian fits (not shown) to the data distributions for $l \in\left[30^{\circ}, 65^{\circ}\right]$ (left plot) and $l \in\left[65^{\circ}, 85^{\circ}\right]$ (middle plot) yield values for the mean consistent with $b=0^{\circ}$ and for $\sigma$ of $0.9^{\circ} \pm 0.3^{\circ}$ and $2.0^{\circ} \pm 0.2^{\circ}$, respectively. Assuming a larger exclusion region around the Galactic plane in the background estimation (see previous section) gives the same narrow width. The emission profiles as predicted by the optimized GALPROP model are overlaid. The blue line shows the total flux prediction, the green the inverse Compton, and the red the pion contribution. For both the GALPROP prediction as well 
as the data the flux numbers become smaller and the distributions become wider farther away from the Galactic center. In order to compare the predicted profiles with the measured profiles, the $\chi^{2}$ in both regions is calculated. In the inner Galaxy region $\left(l \in\left[30^{\circ}, 65^{\circ}\right]\right.$ and $b \in\left[-10^{\circ}, 10^{\circ}\right]$ ) a $\chi^{2}$ of 18.3 with 20 degrees of freedom is derived, corresponding to a probability of $57 \%$ that the $\chi^{2}$ for a correct model exceeds the observed one by chance. In the Cygnus region, this chance probability is calculated to be $10^{-4}$. The discrepancy between the model prediction of the latitude profile and the data in the Cygnus region is investigated further by fitting the measured profile between $b=10^{\circ}$ and $b=-10^{\circ}$ to a linear combination of the predicted pion and IC profiles, $C_{I C} \cdot p_{I C}(b)+C_{\pi} \cdot p_{\pi}(b)$. The factors $C_{I C}$ and $C_{\pi}$ are varied independently between 0.1 and 10 in steps of 0.1 . The minimum value of $\chi^{2}$ is obtained for $C_{\pi}=6.9$, i.e. an increase of the pion contribution with respect to the GALPROP prediction by a factor 6.9 , and $C_{I C}=0.1$. The resulting chance probability is $3 \%$. Performing the same fit to the inner Galaxy latitude profile yields an increase of the pion contribution of $C_{\pi}=5.8$ and a decrease of the $\mathrm{IC}$ contribution of $C_{I C}=0.1$ with respect to the GALPROP prediction. The chance probability of this result is $93 \%$, showing that there is no significant improvement between the two cases.

Figure 3 also shows the Galactic latitude profile for $b \in\left[-10^{\circ}, 10^{\circ}\right]$ in the region above Cygnus $\left(l \in\left[85^{\circ}, 110^{\circ}\right]\right.$, right plot). No significant enhancement near the Galactic plane is visible.

\section{Discussion}

Measurements of the diffuse $\mathrm{TeV} \gamma$-ray flux from the Galactic Plane as well as its spatial distribution, the latitude and longitude profiles, have been presented. The diffuse $\gamma$-ray flux was compared to predictions based on both the conventional and the optimized GALPROP models. In the $l \in\left[30^{\circ}, 65^{\circ}\right]$ range the optimized GALPROP prediction is consistent with the Milagro measurement. According to the GALPROP model the diffuse emission near $15 \mathrm{TeV}$ is dominated by the inverse Compton component, which in turn is dominated by $\sim 100 \mathrm{TeV}$ electrons scattering off the CMB (60 to $70 \%$ of the IC component, see Figure 21). Therefore, Milagro measurements can be interpreted as showing the first indication of the cosmicray spectrum up and beyond $100 \mathrm{TeV}$ using the predictions of the GALPROP optimized model. The propagated average electron spectrum calculated by the optimized GALPROP model predicts a flux of about four times the locally measured flux and extends above 100 $\mathrm{TeV}$ with a differential spectral index of -3 . An alternative explanation would be a harder nucleon injection spectrum in the inner Galaxy than measured locally, but this would have to be checked against local antiproton measurements (Moskalenko et al. 1998; Strong et al. 
2007). Studies of the lateral $\gamma$-ray emission profile in the inner Galaxy show that the profile is rather narrow and suggest a better agreement with the pion assumption. Better sensitivity is needed however to significantly differentiate between the IC and the pion hypotheses.

The Cygnus region is the region with the most significant flux excess in the Milagro spatial maps. The emission from the Cygnus region of the Galaxy at longitudes $l \in\left[65^{\circ}, 85^{\circ}\right]$ shows an excess by a factor of two when compared to the GALPROP optimized model predictions. Also for the Cygnus region, the model predictions are dominated by IC scattering of electrons off the CMB (see Figure 2). However, the measured and predicted profiles are inconsistent (see Figure 3). Decreasing the IC contribution (which has a broad profile distribution) and increasing the pion contribution (which has a narrow profile distribution) with respect to the model prediction improves the agreement in the shape, reflecting that the latitude distribution measured by Milagro is rather narrow compared to the distribution predicted by the optimized GALPROP model.

The Cygnus region hosts intense star formation activity, and is abundant with molecular clouds and candidate cosmic ray sources. The HEGRA source TeV J2032+413 is located in the area with the largest concentration of molecular and atomic hydrogen in the Cygnus region and spatially coincident with the Milagro source MGRO J2031+41 (Abdo et al.|2007a). For TeV J2032+413 an association has been proposed with Cygnus OB2, the largest cluster of more than 2700 identified young stars at 5000 light year distance (Aharonian et al. 2005a; Anchordoqui et al. 2007). Very close to TeV J2032+4130, the VLA has detected a weak non-thermal shell supernova remnant (Butt et al. 2006) and more recent XMMNewton observations have revealed the presence of an extended X-ray source co-located with $\mathrm{TeV}$ J2032+4130, which could be produced by an unknown population of faint X-ray sources (Horns et al. 2007). In order to explain the X-ray and $\mathrm{TeV}$ emissions from $\mathrm{TeV}$ J2032+413, Horns et al. (2007) proposed both a hadronic and a leptonic scenario. In the hadronic scenario, the X-ray emission would be produced by the synchrotron emission of secondary electrons and the emission above $10 \mathrm{TeV}$ should show a hard spectrum. If the $\mathrm{X}$-ray and $\mathrm{TeV}$ emissions were instead of leptonic origin, the spectrum above $10 \mathrm{TeV}$ should be rather soft due to the unavoidable Klein-Nishina suppression of the inverse Compton cross section.

If cosmic rays are injected into the Galactic interstellar medium by any of these candidate counterparts to TeV J2032+4130 and MGRO J2031+41, then the newly accelerated cosmic rays interact with the local gas and produce $\gamma$ rays within $100 \mathrm{pc}$ from the source with spectra which might be significantly different from the average $\gamma$-ray spectrum, because the cosmicray spectrum has not yet been steepened by diffusion (Aharonian \& Atoyan 1996). Assuming a distance of about $1 \mathrm{kpc}$, the extension of the entire Cygnus region is about $300 \mathrm{pc}$, and thus 
a single accelerator might influence strongly the entire region. Assuming the diffusion process to be energy dependent, the emission from a molecular cloud close to such an accelerator could be due to high energy protons which have been accelerated first, have left the remnant first, and have already reached the cloud, as they diffuse faster. Thus this emission would have a flatter spectrum and could produce VHE $\gamma$ rays in the Milagro energy range during the first 10,000 years after the SN explosion Gabici \& Aharonian 2007; Moskalenko et al. $2007 \mathrm{~b})$. Leptonic mechanisms for the production of VHE $\gamma$ rays are disfavored by the fast cooling of such highly energetic electrons. Assuming a cosmic-ray source of total energy $10^{51}$ ergs having exploded less than 10,000 years ago in the ISM and a molecular cloud of total mass greater than $10^{4}$ and less than $10^{5}$ solar masses (Dobashi et al. 1994), the hard spectrum cosmic ray nucleons scattering off the targets in the Cygnus region would produce a flux between $10^{-15}$ and $10^{-14} \mathrm{TeV}^{-1} \mathrm{~cm}^{-2} \mathrm{~s}^{-1}$ near $15 \mathrm{TeV}$ (see Figure 1 of Gabici \& Aharonian (2007)). Calculated from Table 1, the emission measured by Milagro near $15 \mathrm{TeV}$ not explained by GALPROP is roughly $2.9 \times 10^{-14} \mathrm{TeV}^{-1} \mathrm{~cm}^{-2} \mathrm{~s}^{-1}$, meaning that only a few strong young accelerators in the Cygnus region are needed to explain the excess emission measured by Milagro.

The results herein and previously presented by the Milagro Collaboration provide further evidence for the existence of cosmic-ray accelerators in the Cygnus region of the Galaxy, favoring hadronic production mechanisms (Beacom \& Kistler 2007; Anchordoqui et al. 2007; Butt et al. 2006). If the emission originates from hadronic interactions of hard spectrum cosmic rays, a corresponding flux of neutrinos will arise from decay of charged pions, and neutrino detectors such as Icecube could provide the conclusive probe of proton acceleration in the Galaxy (Beacom \& Kistler 2007; Gabici \& Aharonian 2007).

Experiments like GLAST (with its improved sensitivity and angular resolution with respect to EGRET) will be able to separate the truly diffuse $\gamma$-ray emission from a potentially unresolved source component, and probe the spatial distribution of the diffuse emission at $\mathrm{GeV}$ energies (the "GeV excess"). Experiments like the proposed High Altitude Water Cherenkov (HAWC) detector will be able to constantly survey large regions of the sky, in particular the Galactic plane, at $\gamma$-ray energies up to $100 \mathrm{TeV}$ with 10 to 15 times the sensitivity of Milagro. Because of its more southerly location it will also be more sensitive to Galactic plane regions below $l=65^{\circ}$. This will put stricter constraints on models like GALPROP and thus provide crucial information about the propagation of cosmic rays above $100 \mathrm{TeV}$.

We thank Scott Delay, Michael Schneider, and Owen Marshall for their dedicated efforts on the Milagro experiment. We also gratefully acknowledge the financial support of the National Science Foundation (under grants PHY-0245234, -0302000, -0400424, -0504201, - 
0601080, and ATM-0002744), the Department of Energy (Office of High Energy Physics), Los Alamos National Laboratory, the University of California, and the Institute for Geophysics and Planetary Physics at Los Alamos National Laboratory. I. V. Moskalenko acknowledges partial support from the NASA APRA grant.

\section{REFERENCES}

Abdo, A. A., et al. 2007a, ApJ, 658, L33

Abdo, A. A., et al. 2007b, ApJ, 664, L91

Agostinelli, S., et al. 2003, NIM A 506 2003, 250

Aharonian, F. A., \& Atoyan, A. M. 1996, A\&A, 309, 917

Aharonian, F. A., \& Atoyan, A. M. 2000, A\&A, 362, 937

Aharonian, F., et al. 2005a, A\&A, 431, 197

Aharonian, F., et al. 2005b, Science, 307, 1938

Aharonian, F., et al. 2006a, A\&A, 449, 223

Aharonian, F., et al. 2006b, Nature, 439, 695

Aharonian, F., et al. 2006c, ApJ, 636, 777

Amenomori, M., et al. 2006, Science, 314, 439

Anchordoqui, L., Halzen, F., Montaruli, T., \& O’Murchadha, A. 2007, Phys. Rev. D, 76, 067301

Atkins, R., et al. 2003, ApJ, 595, 803

Atkins, R., et al. 2004, ApJ, 608, 680

Atkins, R., et al. 2005, Physical Review Letters, 95, 251103

Baughman, B. M., Atwood, W. B., Johnson, R. P., Porter, T. A., \& Ziegler, M. 2007, ArXiv e-prints, 706, arXiv:0706.0503

Beacom, J. F., \& Kistler, M. D. 2007, Phys. Rev. D, 75, 083001 
Bertsch, D. L., Dame, T. M., Fichtel, C. E., Hunter, S. D., Sreekumar, P., Stacy, J. G., \& Thaddeus, P. 1993, ApJ, 416, 587

Butt, Y. M., Combi, J. A., Drake, J., Finley, J. P., Konopelko, A., Lister, M., Rodriguez, J., \& Shepherd, D. 2006, ArXiv Astrophysics e-prints, arXiv:astro-ph/0611731

Casanova, S., \& Dingus, B. L. 2007, Astroparticle Physics (in press), ArXiv e-prints, 711, arXiv:0711.2753

de Boer, W., Sander, C., Zhukov, V., Gladyshev, A. V., \& Kazakov, D. I. 2005, A\&A, 444, 51

Dobashi, K., Bernard, J.-P., Yonekura, Y., \& Fukui, Y. 1994, ApJS, 95, 419

Gabici, S., \& Aharonian, F. A. 2007, ApJ, 665, L131

Gralewicz, P., Wdowczyk, J., Wolfendale, A. W., \& Zhang, L. 1997, A\&A, 318, 925

Heck, D. et al., Report FZKA 6019, 1998

Horns, D., Hoffmann, A. I. D., Santangelo, A., Aharonian, F. A., \& Rowell, G. P. 2007, A\&A, 469, L17

Hunter, S. D., et al. 1997, ApJ, 481, 205

Konopelko, A., et al. 2007, ApJ, 658, 1062

Moskalenko, I. V., Strong, A. W., \& Reimer, O. 1998, A\&A, 338, L75

Moskalenko, I. V., Digel, S. W., Porter, T. A., Reimer, O., \& Strong, A. W. 2007a, Nuclear Physics B Proceedings Supplements, 173, 44

Moskalenko, I. V., Porter, T. A., Malkov, M. A., \& Diamond, P. H. 2007b, arXiv e-prints, 705, arXiv:0705.3854

Porter, T. A., Moskalenko, I. V., Strong, A. W., Orlando, E., \& Bouchet, L. 2008, ApJ682, 400

Porter, T. A., \& Protheroe, R. J. 1997, Journal of Physics G Nuclear Physics, 23, 1765

Prodanović, T., Fields, B. D., \& Beacom, J. F. 2007, Astroparticle Physics, 27, 10

Stecker, F. W., Hunter, S. D., \& Kniffen, D. A. 2008, Astroparticle Physics, 29, 25 
Strong, A. W., Bloemen, H., Diehl, R., Hermsen, W., \& Schönfelder, V. 1999, Astrophysical Letters Communications, 39, 209

Strong, A. W., Moskalenko, I. V., \& Reimer, O. 2000, ApJ, 537, 763

Strong, A. W., Moskalenko, I. V., Reimer, O., Digel, S., \& Diehl, R. 2004a, A\&A, 422, L47

Strong, A. W., Moskalenko, I. V., \& Reimer, O. 2004b, ApJ, 613, 956

Strong, A. W., Moskalenko, I. V., \& Ptuskin, V. S. 2007, Annu. Rev. Nucl. Part. Sci., 57, 285 

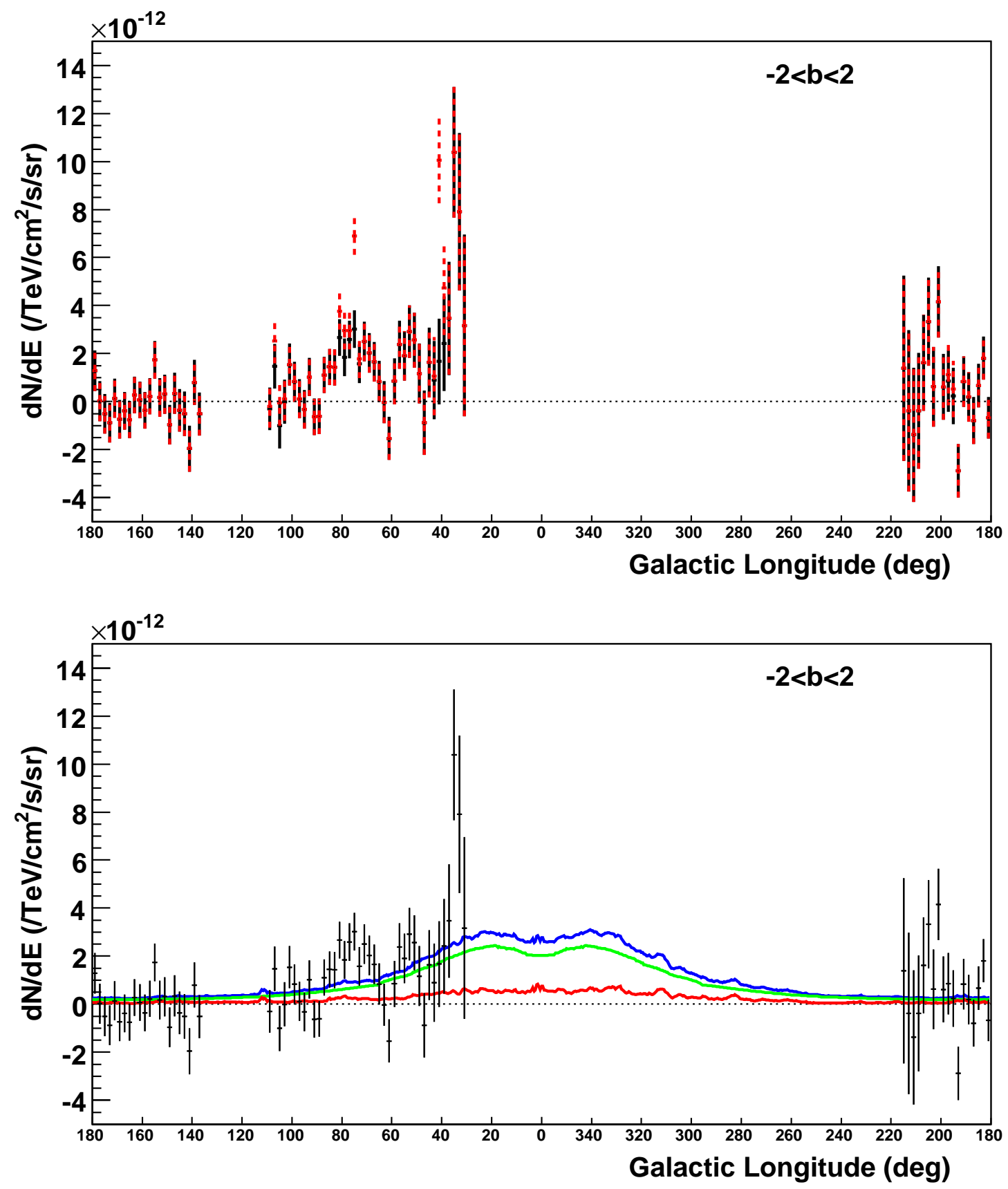

Fig. 1.- Galactic longitude profile of the $\gamma$-ray emission around $15 \mathrm{TeV}$ in the Galactic plane as measured by Milagro. Upper plot: Red data points with dashed error bars - no subtraction of source contributions, black data points - after subtraction of source contributions. Lower plot: Source-subtracted profile overlaid with prediction of the optimized GALPROP model - the red line is the pion contribution, the green line the IC contribution, and the blue line represents the total flux prediction between Galactic latitudes \pm 2 degrees. There are no data points in the region of longitude $l \in\left[-144^{\circ}, 29^{\circ}\right]$ because it is below the Milagro horizon. The region $l \in\left[111^{\circ}, 135^{\circ}\right]$ is excluded because the analysis method is insensitive here (see text for details). 

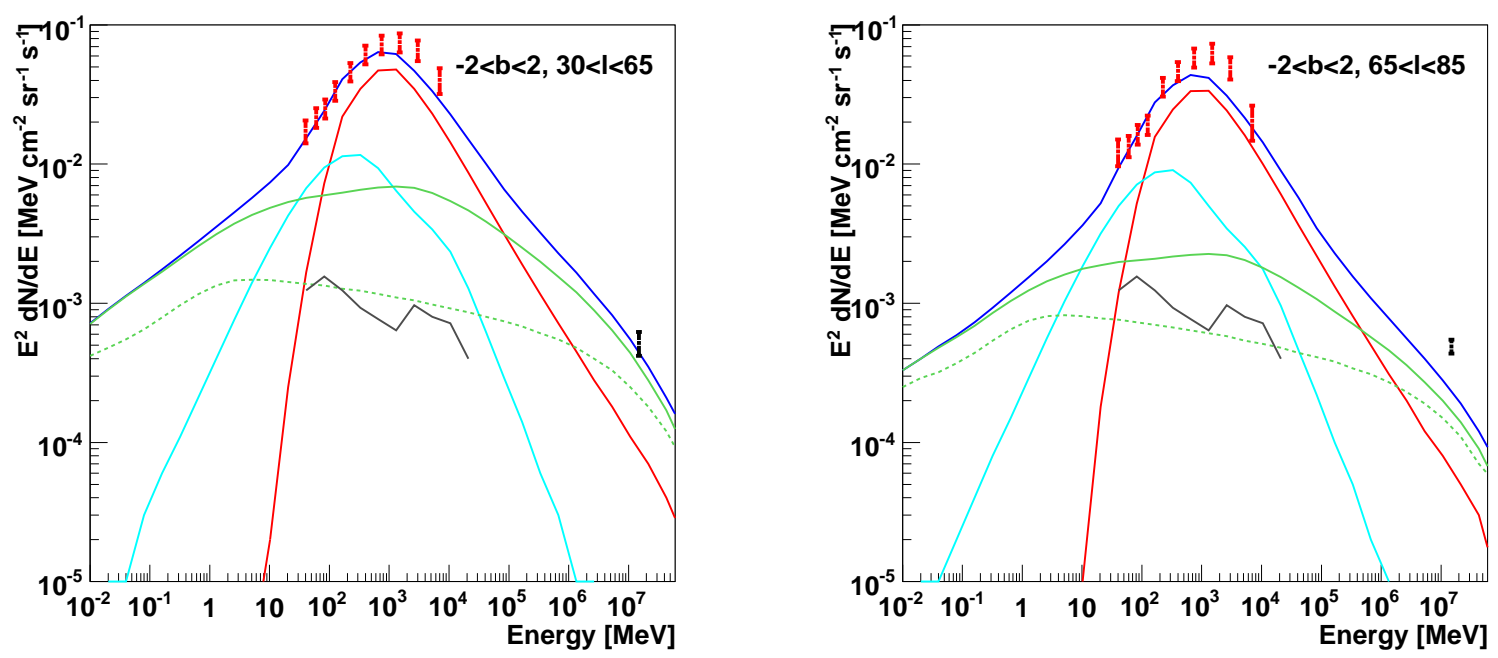

Fig. 2.- Gamma-ray spectra of the diffuse emission as predicted by the optimized GALPROP model for the Galactic plane - left plot: inner Galaxy $\left(l \in\left[30^{\circ}, 65^{\circ}\right]\right)$, right plot: Cygnus region $\left(l \in\left[65^{\circ}, 85^{\circ}\right]\right)$. The red bars represent EGRET data, the black bar the Milagro measurement, where the length of the bar represents the statistical uncertainty only. The dark blue line represents the total diffuse flux predicted by the optimized GALPROP model, the dark gray line the extragalactic background, and the light blue line the bremsstrahlung component. The two contributions at Milagro energies are shown as red line, the pion contribution, and green line, the total IC contribution. The green dashed line shows the dominant IC contribution from scattering of electrons off the cosmic microwave background, which amounts to about 60 to $70 \%$ of the IC component at Milagro energies. Other IC contributions which are less important, such as infrared and optical, are not shown separately. 

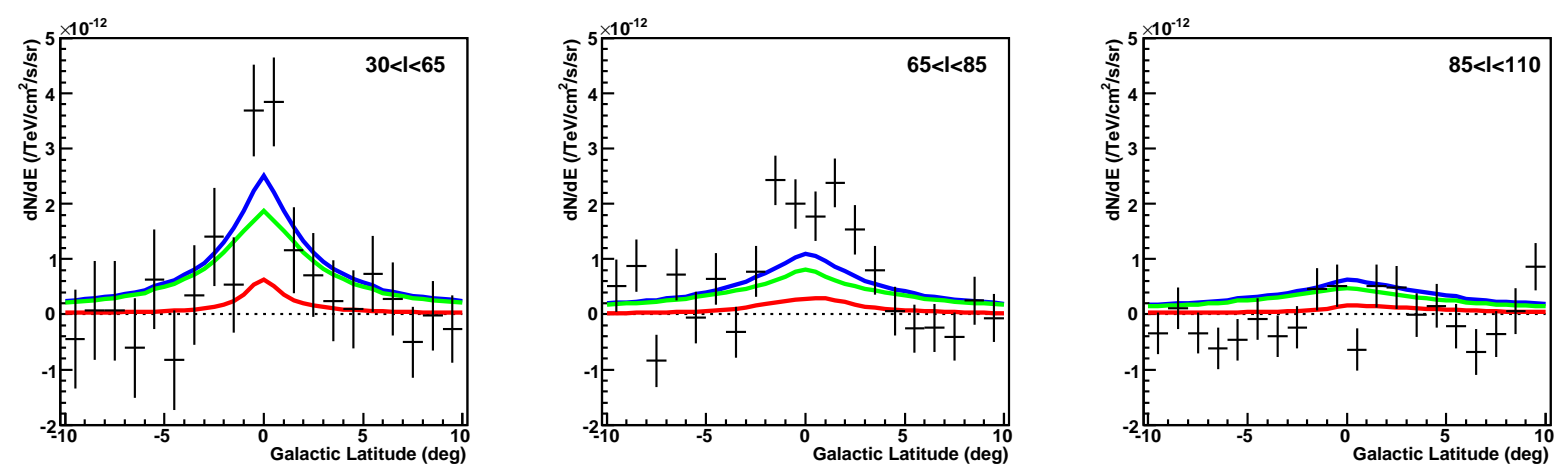

Fig. 3.- Source-subtracted Galactic latitude profile of the $\gamma$-ray emission around $15 \mathrm{TeV}$ in the inner Galaxy (left plot), in the Cygnus region (middle plot), and in the region above Cygnus (right plot) as measured by Milagro (points with errors) and predicted by the optimized GALPROP model. The blue curve is the total $\gamma$-ray flux, the red curve the pion and the green curve the IC contribution. 